\title{
Value of Examination Under Fluoroscopy for the Assessment of Sacroiliac Joint Dysfunction
}

\section{TO THE EditOR:}

It was with great interest that we read the article by Jonathan P. Eskander et al " Value of Examination Under Fluoroscopy for the Assessment of Sacroiliac Joint Dysfunction" published in the 2016 March/July issue of Pain Physician (1).

This is a well-designed prospective article which demonstrates that a positive result was considered as more than 2 hours of greater than $75 \%$ reduction in visual analog scale scores with local anesthesia injection guided by fluoroscopy, the gold standard technique for the diagnosis of sacroiliac joint dysfunction syndrome. The result is satisfactory, which showed significant sensitivity and specificity, in addition to positive and negative predictive values for both the conventional examinations as well as the examination under fluoroscopy. However, we would like to make some suggestions after celebrating the authors for this successful article.

The sacroiliac joint (SIJ) is one of the common sources of chronic low back pain (LBP). Its prevalence varies from $15 \%$ to $30 \%$ based on the clinical evaluation (2). A reference standard for diagnosing SIJ pain was recommended in 1994 by the International Association for the Study of Pain (IASP) (3). IASP's 3 diagnostic criteria were:

- $\quad$ Pain is present in the region of the SIJ

- $\quad$ Stressing the SIJ by clinical tests that are selective for the joint reproduces the patient's pain

- $\quad$ Selectively infiltrating the putatively symptomatic joint completely relieves the patient of the pain.

We suggested that it is necessary to evaluate and plan the treatment with an algorithm all patients who complain spreading to the lower extremity and not spreading of low back pain of SIE induced with reference to these criteria and studies on SIE dysfunction. \{this paragprah does not make sense. Also, SIE needs to be defined\}

Fortin et al (4) generated a pain referral map using contrast medium injection provocation, followed by local anesthetic injection into the sacroiliac joint in 10 asymptomatic volunteers. Fortin et al (5) also evaluated the applicability of a pain referral map as a screening tool for sacroiliac joint dysfunction. We should evaluate with SIE provocation tests all patients that SIEinduced back pain with reference to the areas of the pain distribution map of the study of Fortin et al $(4,5)$. Laslett et al (6) examined the diagnostic power of pain provocation of sacroiliac joint tests singly and in various combinations, in relation to an accepted criterion standard. Sensitivity and specificity for 3 or more of 6 positive sacroiliac joint tests were $94 \%$ and $78 \%$, respectively. They concluded that composites of provocation sacroiliac joint tests are of value in the clinical diagnosis of a symptomatic sacroiliac joint. Three or more out of 6 tests or any of 2 of 4 selected tests have the best predictive power in relation to the results of intraarticular anesthetic block injections. Van der Wurff et al (7) reported that the presence of 3 or more positive provocation tests of patients with SIJ-induced back pain varied between $65 \%$ and $93 \%$. In addition, it has been reported that these tests can be used instead of the invasive diagnostic methods of SIJ for early diagnosis. Similarly, we suggested that a multitest regimen consisting of 6 SIE pain provocation tests (distraction, compression, Gaenslen test, Patrick's sign and thigh thrust test) is a reliable method for evaluating SIE dysfunction.

As noted in this study, in patients who are evaluated by history, physical examination and provocation tests and in cases where the back pain is thought to be due to SIE dysfunction can be planned injection for diagnosis and treatment of intra- and periarticularinduced pain (1). SIJ blocks only provide information about pathologies of joint origin and SJD developıng secondary to pathologies of structures around the joint can be overlooked. For patients who have pain after injection, it should be thought to be the muscles and ligament-derived pain and the SIJ should be assessed by provocation, palpation, and movement tests to determine which direction of the joint is restricted. The weakness and shortness of muscle-ligaments leading to this limitation resulting in SIE dysfunction should be determined and an appropriate physical therapy and exercise program should be planned.

Finally, we would like to thank Jonathan P. Eskan- 
der et al again for their well-designed prospective article.

Dr. Hilal Telli, MD

TC SB SBÜ

Erzurum Bölge Eүitim ve Arastırma Hastanesi

\author{
Atatürk Mahallesi Çat Yolu Caddesi \\ 25040 Yakutiye \\ Erzurum / TURKEY \\ E-mail: dr.hilaltelli@hotmail.com
}

\section{References}

1. Eskander JP, Ripoll JG, Calixto F, Beakley BD, Baker JT, Healy PJ, Gunduz OH, Shi L, Clodfelter JA, Liu J, Kaye AD, Sharma $S$. Value of examination under fluoroscopy for the assessment of sacroiliac joint dysfunction. Pain Physician 2015; 18:E781E786

2. Maigne JY, Aivaliklis A, Pfefer F. Results of sacroiliac joint double block and value of sacroiliac pain provocation tests in 54 patients with low back pain. Spine 1996; 21:1889-92.
3. Merskey H, Bogduk N. Classification of Chronic Pain: Descriptions of Chronic Pain Syndromes and Definitions of Pain Terms. 2nd ed.: IASP Press, Seattle, WA, 1994.

4. Fortin JD, Aprill CN, Ponthieux B, Pier J. Sacroiliac joint: pain referral maps upon applying a new injection/arthrography technique. Part I: Asymptomatic volunteers. Spine (Phila Pa 1976) 1994; 19:1475- 7 1482.

5. Fortin JD, Aprill CN, Ponthieux B, Pier J. Sacroiliac joints: Pain referral maps upon applying a new injection/arthrography technique. Part II: Clinical evaluation. Spine (Phila Pa 1976) 1994; 19:1483-1489.

6. Laslett M, Aprill CN, McDonald B, Young SB. Diagnosis of sacroiliac joint pain: A validity of individual provocation tests and composites of tests. Man Ther 2005; 10:207-218.

7. Van der Wurff P, Hagmeijer RHM, Meyne W. Clinical tests of the sacroiliac joint. A systemic methodological review. Part 1. Man Ther 2000; 5:30-36. 